\title{
Axonal sensorimotor neuropathy in patients with $\beta$-thalassaemia
}

\author{
E Stamboulis, N Vlachou, M Drossou-Servou, P Tsaftaridis, G Koutsis, N Katsaros, \\ E Economou-Petersen, A Loutradi-Anagnostou
}

J Neurol Neurosurg Psychiatry 2004;75:1483-1486. doi: 10.1136/innp.2003.024794

\begin{abstract}
Objective: The purpose of this study was to investigate the prevalence of peripheral neuropathy in patients with $\beta$ thalassaemia.

Methods: Thirty six patients with a mean age of $29.2 \pm 8.2$ years and 17 healthy controls with a mean age of $27.6 \pm 9.1$ were included in this study. Measurements included the neuropathy symptoms score (NSS), the neuropathy disability score (NDS) as well as nerve conduction studies of two motor (ulnar and peroneal) and two sensory (ulnar and sural) nerves of the right limbs.

Results: A mainly sensory axonal polyneuropathy was present in 19 out of 36 patients (52.7\%). Eight out of these 19 patients also had abnormal NDS values. The neuropathy correlated significantly with the age of the patients and the hematocrit. However, it did not correlate with the presence of antibodies against HCV, the ferritin levels, or with a history of transfusions, desferrioxamine treatment, or splenectomy.

Conclusions: This study showed a high prevalence of a predominantly sensory neuropathy in patients with $\beta$ thalassaemia. The electrophysiological data suggest that the underlying pathology is an axonopathy. Chronic hypoxia of the nerves resulting from severe anaemia may contribute to the pathogenesis of this neuropathy.
\end{abstract}

$\mathrm{T}$ halassaemia comprises a heterogeneous group of hereditary disorders characterised by a decrease in the production of one or more globin chains. Multiple complications can result from chronic anaemia on one hand to transfusion related haemosiderosis on the other. The peripheral nervous system, however, is rarely affected. Myopathic syndromes ${ }^{1-3}$ and peripheral neuropathy have been reported. ${ }^{4-6}$ Desferrioxamine (DFO), used as chelation therapy to reduce transfusion induced iron overload, can cause ophthalmological disorders and high frequency sensorineural hearing loss. ${ }^{7-9}$ Sensorimotor neurotoxicity, associated with high dose DFO treatment has also been reported..$^{10}$

In this study we evaluated patients with $\beta$-thalassaemia clinically and electrophysiologically, and investigated whether factors such as age, clinical severity of thalassaemia (homozygous $\beta$ or intermedia), antibodies against hepatitis $C$ virus $(\mathrm{HCV})$, ferritin levels, haematocrit $(\mathrm{Ht})$, and history of transfusions, splenectomy, or DFO treatment are associated with abnormal findings.

\section{PATIENTS AND METHODS}

We examined a consecutive series of 36 patients with thalassaemia ( 19 women, 17 men; mean age $29.2 \pm 8.2$ years, range 16-58) followed up at two tertiary referral centres.
Patients with diabetes or other known possible causes of neuropathy were excluded from the study. Thirty two had homozygous $\beta$-thalassaemia and four $\beta$-thalassaemia intermedia. All patients had normal vitamin $\mathrm{B}_{12}$, folic acid, and serum protein electrophoresis and immunofixation. No patient had clinical evidence of visual or auditory dysfunction. The control group consisted of 17 healthy individuals ( 12 women, 5 men; mean age $27.6 \pm 9.1$ years, range $16-55$ ).

In all patients the electrophysiological studies were performed by the same investigator. Standard procedures were used. Motor nerve conduction studies (NCS) of the right ulnar and peroneal nerves were performed. Surface electrode recordings were obtained from the abductor digiti minimi and the extensor digitorum brevis. Antidromic sensory nerve conduction studies of the right ulnar and sural nerves were performed. Measurements were taken at temperatures of $32{ }^{\circ} \mathrm{C}$ and $29{ }^{\circ} \mathrm{C}$ for the upper and lower limbs, respectively. Deviations of 2 standard deviations (SD) or more relative to the mean values of the controls were considered abnormal.

The neuropathy was assessed clinically by the neuropathy symptoms score (NSS) and the neuropathy disability score (NDS). ${ }^{11}$ Serum ferritin levels were assessed at the same time. The haematocrit used in the statistical analysis was the mean of the last two bimonthly values before electrophysiological studies. The patients were finally divided into groups depending on history of DFO treatment, blood transfusions, or splenectomy and on the presence or absence of antibodies against HCV. The results were analysed statistically with the two-sample $t$-test of unequal variance and the $\chi^{2}$ test.

\section{RESULTS}

Nine out of 36 patients reported symptoms that could be attributed to neuropathy such as numbness, tingling, hyperpathia, cramps, muscular weakness, muscle tenderness, and restless legs. In eight patients neurological signs of neuropathy were demonstrated, such as reduction in vibration sense, hypoaesthesia of the feet, weakness of the extensor digitorum brevis, and reduced knee and achillean reflexes.

A total of 19 patients $(52.7 \%)$ had pathological values in the nerve conduction studies (table 1). The most frequent abnormal finding was a reduction in the amplitude of the sensory action potential (SAP) of the sural nerve followed by a reduction in the amplitude of compound muscle action potential (CMAP) of the peroneal nerve. Further statistical analysis revealed a significant reduction in the amplitude of the SAP of the sural nerve (patients $17.25 \pm 7.81$, controls $24.40 \pm 6.10, \mathrm{p}<0.01$ ), the amplitude of the CMAP (patients

Abbreviations: CMAP, compound muscle action potential; DFO, desferrioxamine; Ht, haematocrit; NCS, nerve conduction studies; NDS, neuropathy disability score; ns, not significant; NSS, neuropathy symptoms score; SAP, sensory action potential; $S D$, standard deviation 


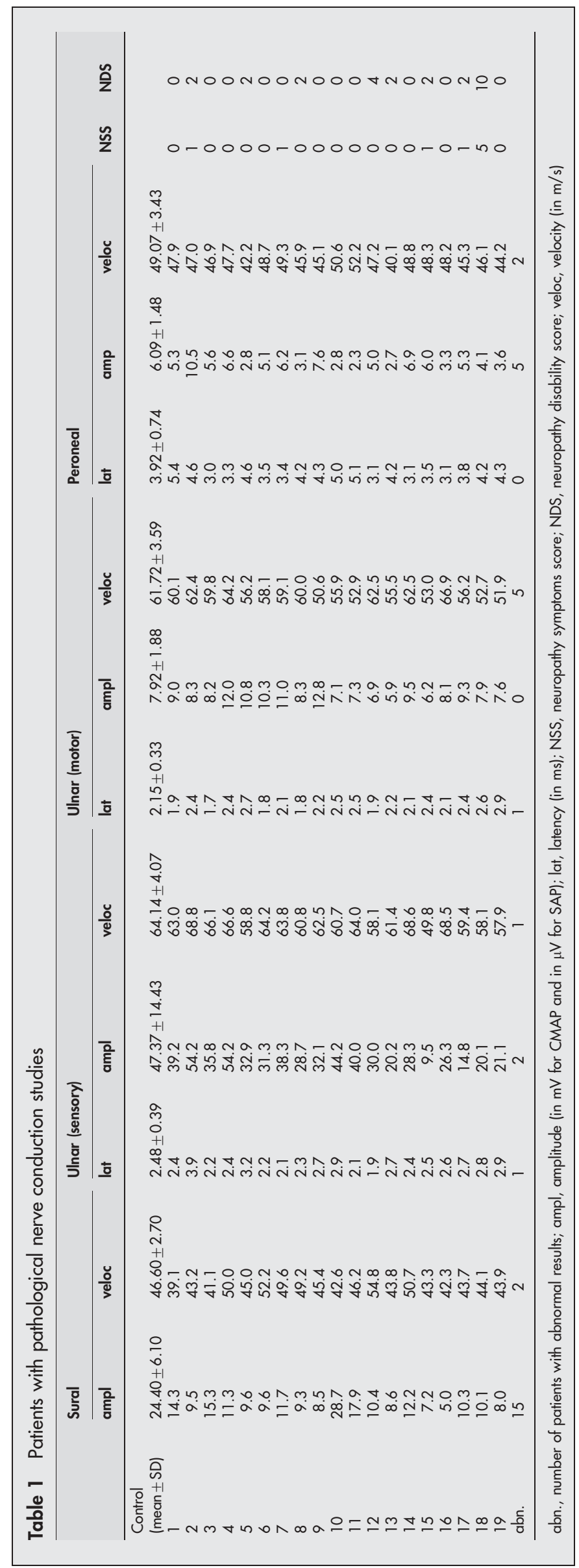


$9.79 \pm 2.53$, controls $7.92 \pm 1.88, \mathrm{p}<0.01)$ and the motor velocity of the ulnar nerve (patients $59.29 \pm 3.61$, controls $61.72 \pm 3.59, p<0.05)$, a prolonged distal motor latency of the ulnar nerve (patients 2.50 \pm 0.36 , controls $2.15 \pm 0.33$, $\mathrm{p}<0.01)$. A reduction in the amplitude of the SAP of the same nerve showed a tendency toward statistical significance (patients 36.47 \pm 13.39 , controls $47.37 \pm 14.43, \mathrm{p}<0.1$ ).

Results of the NCS were further correlated with other clinical parameters. Statistical analysis showed that abnormal findings were more frequent in patients more than 20 years old (21-30: 12/21 patients with abnormal NCS, $\mathrm{p}<0.05$, 31-40: 4/8 with abnormal NCS, p<0.1, 4l-58: 3/3 with abnormal NCS, $\mathrm{p}<0.01$ ) compared with patients less than 20 years old (0/4 with abnormal NCS); in patients with Ht less than 30 (Ht<30: 15/24 with abnormal NCS, Ht>30: 4/12 with abnormal NCS, $\mathrm{p}<0.01$ ); and in patients with abnormal NDS (abnormal NDS: 8/8 with abnormal NCS, normal NDS: 11/28 with abnormal NCS, $\mathrm{p}<0.01$ ). On the other hand, patients with a history of transfusions, DFO administration, or splenectomy were equally likely to have abnormal electrophysiological results as those with no such history (transfusion history: 14/28 with abnormal NCS, no transfusion history: $5 / 8$ with abnormal NCS, $\chi^{2}=0.37$ not significant (ns); receiving DFO: $15 / 28$ with abnormal NCS, not receiving DFO: $4 / 8$ with abnormal NCS, $\chi^{2}=0.02$ ns; splenectomy: 12/9 with abnormal NCS, no splenectomy: 7/17 with abnormal NCS, $\left.\chi^{2}=1.76 \mathrm{~ns}\right)$. The clinical severity of the disease (intermediate or major) did not appear to correlate with the frequency of abnormal findings (homozygous- $\beta$ : 17/32 with abnormal NCS, intermedia: $2 / 4$ with abnormal NCS, $\left.\chi^{2}=0.01 \mathrm{~ns}\right)$. Furthermore, the presence of antibodies against HCV or the presence of neuropathy symptoms was not associated with a significant increase in the frequency of pathological NCS $(\mathrm{HCV}(+)$ : 4/7 with abnormal NCS, HCV $(-): 15 / 29$ with abnormal NCS, $\chi^{2}=0.05$ ns; abnormal NSS: 5/9 with abnormal NCS, normal NSS: 14/27 with abnormal NCS, $\chi^{2}=0.03$ ns). Patients with normal NCS had a mean \pm SD value of ferritin of $2054.1 \pm 1876$ and those with abnormal studies $2426.5 \pm 2355$ ( $t$-test $=0.52 \mathrm{~ns})$.

\section{DISCUSSION}

The NCS in the present report demonstrated that more than half of the patients with $\beta$-thalassaemia $(52.7 \%)$ have evidence of a polyneuropathy. In eight out of the 19 patients with electrophysiologically established polyneuropathy, clinical signs of neuropathy were also present. The findings, as portrayed in table 1, suggest that the neuropathy is axonal and primarily sensory. The neuropathy first affected the lower limbs since pathological values were never limited to the upper limbs of any patient.

It has been reported in previous studies that the prevalence of polyneuropathy in patients with $\beta$-thalassaemia is much lower than our results seem to suggest, ranging from $21 \%$ to $25 \% .^{4-6}$ This difference could be attributed to the fact that the patients in these studies were in the second decade of their life, whereas our patients were mostly in the third and fourth decades. The significant increase in neuropathy with age observed in our study has also been demonstrated in a similar Greek study in the past. ${ }^{4}$ In common with Papanastasiou et al, we found a significant increase in the frequency of neuropathy in patients with a low $\mathrm{Ht}$ and a lack of correlation with ferritin levels, administration of DFO, splenectomy, and transfusions. In addition, we found a lack of correlation of neuropathy with presence of antibodies against HCV. Our finding that severity of disease (homozygous $v$ intermedia) does not seem to correlate with the frequency of abnormal findings is probably due to the fact that the average age of patients with thalassaemia intermedia was greater than the average age of the rest of our patients $(37.0 \pm 12.7$ and $27.8 \pm 6.5$, respectively).

A further interesting finding was that electrophysiologically established neuropathy correlated statistically with the presence of clinical signs of neuropathy, but not with the presence of clinical symptoms. It is possible that nerve hypoxia may lead to symptoms such as numbness, tingling, or cramps without causing any neural tissue damage.

Levine et $a l^{10}$ suggested that DFO can cause sensorimotor neurotoxicity in high doses. They reported two such cases. One patient had asymmetrical neuropathy of the upper limbs with electrophysiological examination showing a radiculopathy and the second patient who was diabetic developed a polyneuropathy. In both cases the neuropathy might not have been due to the administration of DFO.

Wong et $a l^{5}$ also attributed the subclinical neuropathy of their patients (seven out of 34 had pathological sensory conduction velocity of whom three had diabetes) to the long use of DFO but a statistically significant correlation with the dosage of DFO or the duration of its administration was not found. These findings cannot support the view that neuropathy in patients with $\beta$-thalassaemia is due to toxicity of DFO.

The role of chronic ischaemia in the development of neuropathy has been demonstrated both in experimental studies and in clinical situations where ischaemia or hypoxia is present. The nerve ischaemia usually results in axonal damage. The large sensory myelinated fibres seem to be more severely affected. ${ }^{12}$ A high frequency of axonal neuropathy has been found in patients with chronic obstructive pulmonary disease, significantly higher in hypoxic groups. ${ }^{13-16}$ A mainly sensory axonal neuropathy probably due to the endoneural ischaemia, has been found in polycythemia rubra vera, ${ }^{17}{ }^{18}$ peripheral arterial disease ${ }^{19-22}$ and hereditary disorders of haemostasis. ${ }^{23}$ In accordance with the above, the predominantly sensory axonal polyneuropathy found in our patients could also be attributed to chronic hypoxia of the nerves resulting from longstanding anaemia. The higher prevalence of neuropathy in older patients and in patients with a low $\mathrm{Ht}$ is in agreement with this hypothesis.

\section{Authors' affiliations \\ E Stamboulis, N Vlachou, G Koutsis, N Katsaros, Department of Neurology, University of Athens "Aeginition" Hospital, Athens, Greece M Drossou-Servou, E Economou-Petersen, Thalassaemia Unit, General Hospital of Piraeus "Ag.Panteleimon", Athens, Greece P Tsaftaridis, A Loutradi-Anagnostou, Center for Thalassaemia, "Laikon" General Hospital, Athens, Greece \\ Competing interests: none declared}

Correspondence to: E Stamboulis, "Aeginition" Hospital, 74 Vas. Sophias Avenue, GR-1 1528, Athens, Greece; gkoutsi2@otenet.gr

Received 31 July 2003

In revised form 3 December 2003

Accepted 5 December 2003

\section{REFERENCES}

1 Logothetis J, Constantoulakis M, Economidou J, et al. Thalassaemia major (homozygous beta-thalassaemia). A survey of 138 cases with emphasis on neurologic and muscular aspects. Neurology 1972;22:294-304.

2 Mollica F, Romeo MA, di Gregorio F, et al. Neurological and muscular manifestations of thalassaemia major. Arch Fr Pediatr 1980;37:173-5.

3 Shapira Y, Glick B, Finsterbush A, et al. Myopathological findings in thalassaemia major. Eur Neurol 1990;30:324-7.

4 Papanastasiou D, Papanikolaou D, Magiakou AM, et al. Peripheral neuropathy in patients with $b$-thalassaemia. J Neurol Neurosurg Psychiatry 1991;54:997-1000.

5 Wong V, Li A, Lee ACW. Neurophysiologic study of b-thalassaemia patients. J Child Neurol 1993;8:330-5. 
6 Zafeiriou DI, Kousi AA, Tsantali CT, et al. Neurophysiologic evaluation of long-term desferrioxamine therapy in beta-thalassaemia patients. Pediatr Neurol 1998; 18:420-4.

7 Boelaert JR, de Locht M. Side-effects of desferrioxamine in dialysis patients. Nephrol Dial Transplant 1993;(Suppl 1):43-6.

8 Giardina P, Grady RW. Chelation therapy in b-thalassaemia: the benefits and limitations of desferrioxamine. Semin Hematol 1995;32:304-12.

9 Kontzoglou G, Koussi A, Tsatra J, et al. Sensorineural hearing loss in children with thalassaemia major in Northern Greece. Int J Pediatr Otorhinolaryngol 1996:35:223-30.

10 Levine JE, Cohen A, MacQueen M, et al. Sensorimotor neurotoxicity associated with high-dose deferoxamine treatment. J Pediatr Hematol Oncol 1997; 19:139-41.

11 Dyck PJ, Sherman WR, Hallcher LM, et al. Human diabetic endoneuria sorbitol fructose and myoinositol related to sural nerve morphometry. Ann Neurol 1980;8:590-6

12 Teunissen LL, Notermans NC, Wokke JHJ. Relationship between ischemia and neuropathy. Eur Neurol 2000;44:1-7.

13 Nowak D, Bruch M, Arnaud F, et al. Peripheral neuropathies in patients with chronic obstructive pulmonary disease: a multicenter prevalence study. Lung 1990;168:43-51.

14 Pfeiffer G, Kunze K, Bruch M, et al. Polyneuropathy associated with chronic hypoxaemia: prevalence in patients with chronic obstructive pulmonary disease. J Neurol 1990;237:230-3.
15 Bone G Ladurner G, Rolke $M$, et al. Peripheral nerve dysfunction in chronic obstructive pulmonary disease. In: Asbury AK, Bud H, Sleoga E, eds. Sensory Neuropathies. New York: Springer, 1995:143-50.

16 Ozge A, Atis S, Sevim S. Subclinical peripheral neuropathy associated with chronic obstructive pulmonary disease. Electromyogr Clin Neurophysiol 2001;41:185-91.

17 Yiannikas C, McLeod JG, Walsh JC. Peripheral neuropathy associated with polycythemia vera. Neurology 1983;33:139-43.

18 Poza JJ, Cobo AM, Marti Masso JF. Peripheral neuropathy associated with polycythemia vera. Neurologia 1996;1:276-9.

19 England JD, Regensteiner JG, Ringel SP, et al. Muscle denervation in peripheral arterial disease. Neurology 1992;42:994-9.

20 Nukada H, van Rij AM, Packer SG, et al. Pathology of acute and chronic ischaemic neuropathy in atherosclerotic peripheral vascular disease. Brain 1996;119:1449-60.

21 Papapetropoulou V, Tsolakis J, Terzis S, et al. Neurophysiologic studies in peripheral arterial disease. J Clin Neurophysiol 1998; 15:447-50

22 Weinberg DH, Simovic D, Isner J, et al. Chronic ischemic monomelic neuropathy from critical limb ischemia. Neurology 2001;57:1008-12.

23 Stamboulis E, Syrigou-Papavasiliou A, Platokouki HG, et al. Subclinical neuropathy in children with inherited haemostasis disorders. Electromyogr Clin Neurophysiol 1992;32:511-14. 